Family Profile No. 08, 2018

\title{
Characteristics of First-Time Mothers Aged 40 and Older
}

Author: Karen Benjamin Guzzo

As childbearing has been increasingly delayed, birth rates for women aged 40 and older have increased. The majority of births to mothers 40 and older are second or later births, however, about a fifth of such births are first births. Little is known about firsttime mothers 40 and older or how they compare to mothers who began childbearing earlier. This profile uses the 2016 Current Population Survey June Fertility Supplement to compare the characteristics of mothers aged 40-65 among those who began childbearing prior to age 40 and those who began childbearing at age 40 or older. Of mothers $40-65$, only $2.1 \%$ had their first birth after age 40 , representing about 400,000 women.

\section{Union Status and Age at First Birth}

- Among all mothers aged $40-65$ in 2016 , the majority (about three fourths) reported being married at their first birth.

- Cohabiting at first birth was more common among mothers who began childbearing prior to age 40 (14\%) than among mothers who began childbearing later (8\%).

- About one in six mothers (17\%) who began childbearing at 40 or later was single at first birth, whereas one in eight mothers (12\%) who began childbearing earlier was neither married nor cohabiting.

- In 2016, there were about 66,000 single mothers between the ages of 40 and 65 who had their first child at age 40 or older.
Figure 1. Union Status at First Birth among Mothers 40-65 by Age at First Birth, 2016

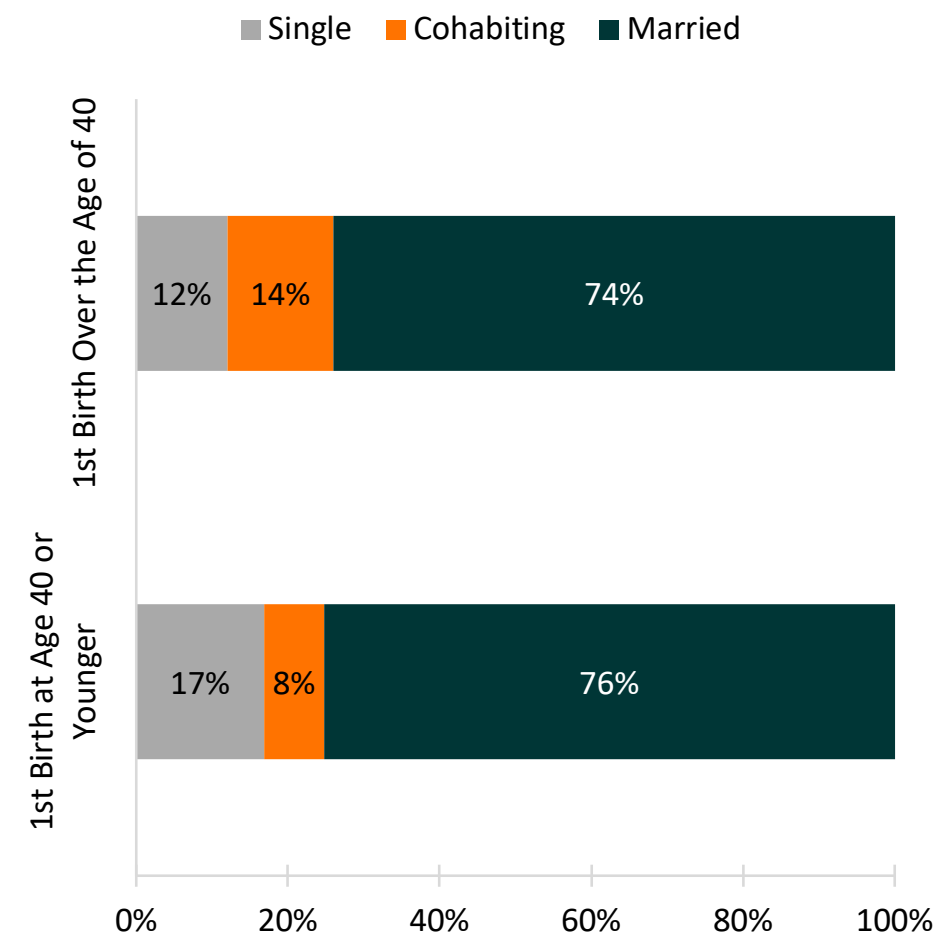

Source: NCFMR analyses of CPS June Fertility Supplement, 2016

\section{Of mothers 40-65, only 2.1\% had their first birth after age 40, representing about 400,000 women.}


Education and Age at First Birth

- Mothers who delayed childbearing until age $\mathbf{4 0}$ or later were better educated than their peers who entered into parenthood earlier.

- Fifty-six percent of older first-time mothers had at least a Bachelor's degree compared to $36 \%$ of mothers who began childbearing earlier.

Figure 2. Education among Mothers 40-65, by Age at First Birth, 2016

$\square$ <H.S. $\quad$ H.S./GED $\quad$ Some College $\square$ Bachelor's+

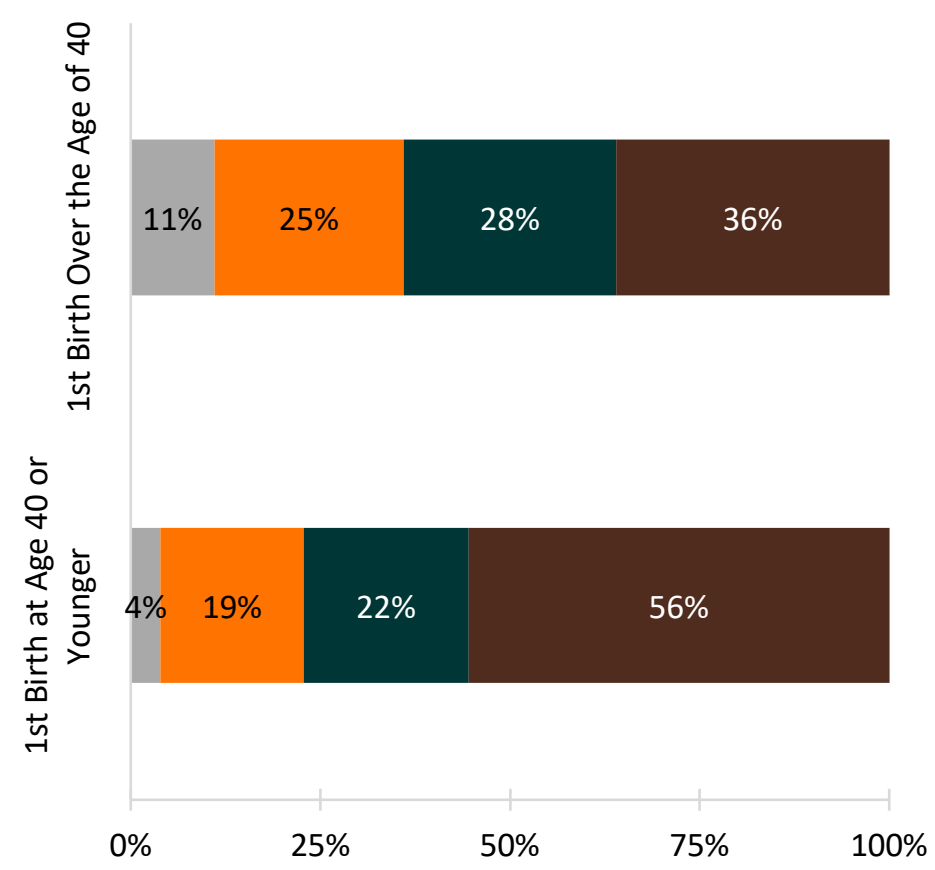

Source: NCFMR analyses of CPS June Fertility Supplement, 2016
Race-Ethnicity and Age at First Birth

- Slightly more mothers who had their first birth at age 40 or older were White compared to those who had their first birth prior to age 40 (64\% and $60 \%$, respectively).

- Nearly twice as many mothers who began childbearing prior to age 40 were Hispanic (20\%) compared to mothers who delayed childbearing (11\%).

- Asians comprised a larger percentage of older firsttime mothers (12\%) than mothers who began childbearing younger than age 40 (8\%).

Figure 3. Race/Ethnicity among Mothers 40-65, by Age at First Birth, 2016

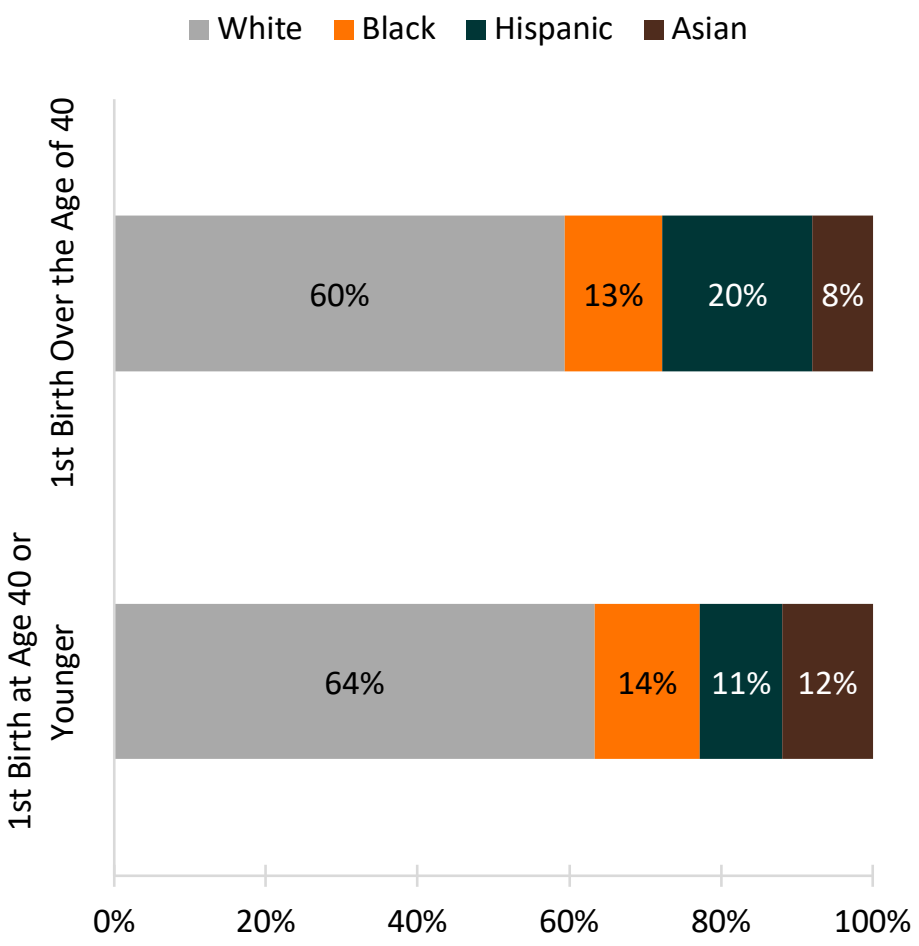

Source: NCFMR analyses of CPS June Fertility Supplement, 2016

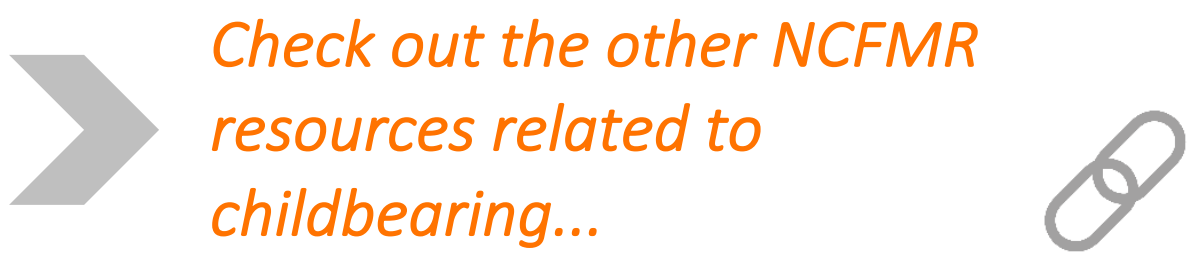

Data Source:

Sarah Flood, Miriam King, Steven Ruggles, and J. Robert Warren. Integrated Public Use Microdata Series, Current Population Survey: Version 5.0. [dataset]. Minneapolis: University of Minnesota, 2017. https://doi.org/10.18128/D030.V5.0.

\section{Suggested Citation:}

Guzzo, K. B. (2018). Characteristics of First-Time Mothers Aged 40 and Older. Family Profiles, FP-18-08. Bowling Green, OH: National Center for Family \& Marriage Research. https://doi.org/10.25035/ncfmr/fp-18-08.

\section{BGSU. National Center for Family \& Marriage Research}

http://www.bgsu.edu/ncfmr ncfmr@bgsu.edu (419) 372-3119 